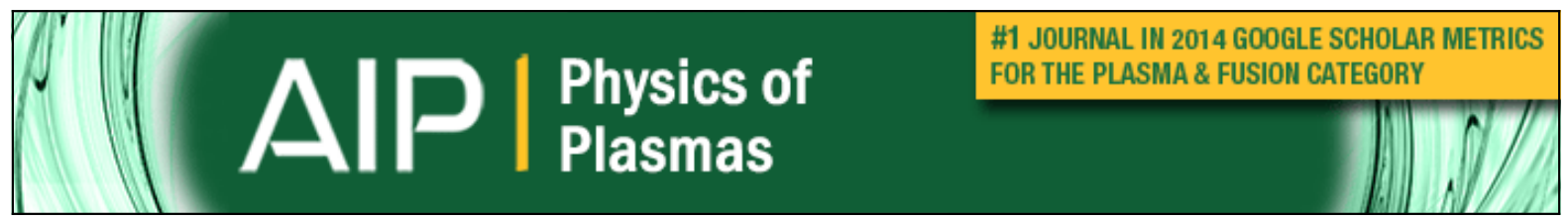

\title{
Measurements of the runaway electron energy during disruptions in the tokamak TEXTOR
}

M. Forster, K. H. Finken, M. Lehnen, O. Willi, Y. Xu, and TEXTOR Team

Citation: Physics of Plasmas 19, 052506 (2012); doi: 10.1063/1.4717759

View online: http://dx.doi.org/10.1063/1.4717759

View Table of Contents: http://scitation.aip.org/content/aip/journal/pop/19/5?ver=pdfcov

Published by the AIP Publishing

\section{Articles you may be interested in}

Mechanism of runaway electron beam formation during plasma disruptions in tokamaks

Phys. Plasmas 22, 040704 (2015); 10.1063/1.4919253

Energetics of runaway electrons during tokamak disruptions

Phys. Plasmas 19, 012507 (2012); 10.1063/1.3671974

Poloidal beta and internal inductance measurement on HT-7 superconducting tokamak

Rev. Sci. Instrum. 78, 093501 (2007); 10.1063/1.2779213

Effect of the vacuum vessel on magnetic measurements in TCABR tokamak

Rev. Sci. Instrum. 75, 5038 (2004); 10.1063/1.1807001

Dynamics of high energy runaway electrons in the Frascati Tokamak Upgrade

Phys. Plasmas 10, 2350 (2003); 10.1063/1.1574328

\section{Did your publisher get}

18 MILLION DOWNLOADS in 2014?

AIP Publishing did.

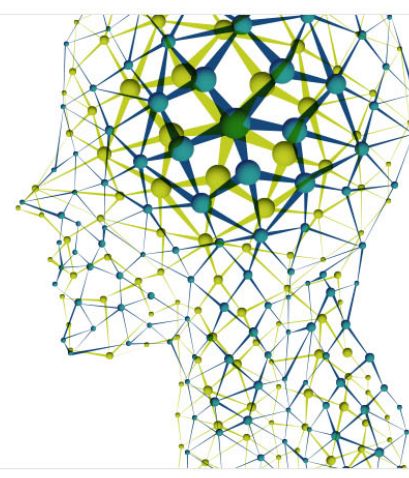




\title{
Measurements of the runaway electron energy during disruptions in the tokamak TEXTOR
}

\author{
M. Forster, ${ }^{1, a)}$ K. H. Finken, ${ }^{1}$ M. Lehnen, ${ }^{2}$ O. Willi, ${ }^{1} \mathrm{Y} . \mathrm{Xu},{ }^{3}$ and the TEXTOR Team ${ }^{2}$ \\ ${ }^{1}$ Institut für Laser- und Plasmaphysik, Heinrich-Heine-Universität Düsseldorf, Universitätsstr. 1, \\ 40225 Düsseldorf, Germany \\ ${ }^{2}$ Institut für Energie- und Klimaforschung, Forschungszentrum Jülich GmbH, EURATOM Association, \\ 52425 Jülich, Germany \\ ${ }^{3}$ Laboratory for Plasma Physics, Ecole Royale Militaire-Koninklijke Militaire School, \\ Avenue de la Renaissance 30, 1000 Brussels, Belgium
}

(Received 27 January 2012; accepted 22 April 2012; published online 18 May 2012)

Calorimetric measurements of the total runaway electron energy are carried out using a reciprocating probe during induced TEXTOR disruptions. A comparison with the energy inferred from runaway energy spectra, which are measured with a scintillator probe, is used as an independent check of the results. A typical runaway current of $100 \mathrm{kA}$ at TEXTOR contains 30 to $35 \mathrm{~kJ}$ of runaway energy. The dependencies of the runaway energy on the runaway current, the radial probe position, the toroidal magnetic field and the predisruptive plasma current are studied. The conversion efficiency of the magnetic plasma energy into runaway energy is calculated to be up to 26\%. (C) 2012 American Institute of Physics. [http://dx.doi.org/10.1063/1.4717759]

\section{INTRODUCTION}

A tokamak discharge, which leaves the stable operational space, may end in a violent interruption of the plasma current-a disruption. $^{1-3}$ This instability is commonly described by two major phases. Within about $1 \mathrm{~ms}$ the magnetic confinement is lost and the thermal energy of the plasma is expelled to the plasma facing components (PFCs) in form of heat loads. Due to this thermal quench, the plasma cools down rapidly. During the second phase of the disruption, the magnetic energy of the plasma is released. The current quench can last up to several hundred milliseconds. A significant increase of the plasma resistivity and the voltage around the torus lead to the formation of large electric fields which can accelerate the plasma electrons. As the collision frequency of the electrons is a strongly decreasing function of their velocity, the electrons can run away and reach velocities in the relativistic regime. These runaway electrons (REs) (Ref. 4) can easily gain several tens of MeV from the magnetic plasma energy. The practically collisionless REs retard the decay of the plasma current. Eventually, the RE current plateau is depleted and the REs leave the plasma in sharp bursts which were first observed via X-ray diagnostics. $^{2,5}$ The bursts are around $0.1 \mathrm{~ms} \mathrm{long}^{6}$ and they strike the PFCs on areas of a few tens of square centimeters. The energetic REs can propagate through several centimeters of low atomic number material like graphite or beryllium. Underlying high atomic number materials like tungsten or copper can be molten which is especially relevant for actively cooled PFCs. ${ }^{7}$ Consequently, the generation of substantial RE currents during disruptions can reduce the lifetimes of PFCs significantly or even cause instant damages. ${ }^{8}$

The reactor size tokamak ITER (Ref. 9) is predicted to be inherently susceptible for the generation of RE currents

\footnotetext{
${ }^{\text {a)} E l e c t r o n i c ~ m a i l: ~ M i c h a e l . F o r s t e r @ u n i-d u e s s e l d o r f . d e . ~}$
}

during disruptions. ${ }^{10}$ The conversion of up to $70 \%$ of the predisruptive plasma current into a RE current will lead to the generation of REs which carry about $50 \mathrm{MJ}$. Due to the small strike areas, this will cause peak energy depositions high enough to melt or ablate the ITER wall materials tungsten, beryllium and graphite. Thus, REs are a concern for the integrity of ITER and studies of the RE energy are needed to enable estimations of the hazard potential.

REs in disruptions have been studied using several detection methods. The X-rays produced by REs which strike PFCs, ${ }^{5,11-13}$ neutrons which result from photonuclear reactions, ${ }^{11,14}$ the electron cyclotron emission ${ }^{15,16}$ and the synchrotron radiation ${ }^{17-19}$ of the REs can be measured. Additionally, specialized probes are used to detect the REs at the plasma edge. ${ }^{20-22}$ In order to mitigate the generation of REs, several techniques are studied. Massive gas injection $^{23-26}$ and pellet injection ${ }^{27,28}$ aim at the suppression of the RE generation due to a steep increase of the electron density. In the case of gas, typically more than $10^{22}$ atoms must be injected. The resulting densities are expected to take the vacuum systems of large tokamaks to their operational limits. Magnetic perturbations, provided by external field coils, ${ }^{16,29-31}$ are studied as a tool to increase the RE transport and hence to decrease the lifetimes and the energy gain of the REs. The fast injection of a moderate amount of gas is proposed for studies at Tore Supra and TEXTOR. ${ }^{32,33}$ Resulting magnetic turbulences are believed to increase the RE transport significantly.

The field of RE research has both, a long tradition and a great importance. Nevertheless, measurements of the RE energy in tokamak disruptions are rare. The RE energy content of JET disruptions is inferred from the temperature increase of hot spots. ${ }^{34}$ Measurements of the RE energy are mandatory to calculate the conversion effeciency of magnetic plasma energy into RE energy. This variable often appears as an input parameter for predictive RE models. 
In Sec. II of this paper, the development of a probe, designed to withstand tokamak disruptions and to measure the RE energy by calorimetry, is presented. Sec. III contains the description of a measurement campaign with the RE calorimeter probe at the tokamak TEXTOR. The experimental results are given and discussed in Sec. IV. Finally, the most important achievements are summarized.

\section{RUNAWAY CALORIMETER PROBE}

The calorimeter probe is designed to get struck by a disruption generated RE beam. The electrons deposit their energy in the probe material and consequently heat it. The resulting temperature profile flattens within several tens of seconds and an equilibrium temperature establishes throughout the material. This value of the temperature can be measured and used to estimate the RE energy in the disruption. The probe must be robust enough to withstand the disruption heat load and the impact of the RE beam. The REs have to get stopped in the probe material without melting it. Hence, a high electron stopping power, a high melting temperature and robustness in the face of thermal shocks are mandatory for the probe material.

In order to find the optimal choice, tests were carried out using the Geant4 code (Refs. 35 and 42) to simulate the energy deposition of a RE beam in different materials. ${ }^{7}$ Geant4 is an established toolkit which was developed at CERN for 3D simulations of elementary particles, their interaction with matter and the response of a detector. User defined primary particles are followed stepwise through the detector material. For each step the loss of the particles' energy and the generation of daughter particles are calculated. This is done for all primary and daughter particles in the defined volume. For the test simulations a RE beam with an exponential energy spectrum (decay parameter $\lambda_{E}=0.125$ $\mathrm{MeV}^{-1}$ ) and an exponential radial distribution (decay parameter $\lambda_{r}=0.085 \mathrm{~mm}^{-1}$ ) was chosen based on the results of former RE probe measurements. ${ }^{21}$ A RE beam, which carries a current of $100 \mathrm{kA}$, is typical for TEXTOR (Ref. 29) and it was assumed to fully strike the probe. A toroidal magnetic field of $B_{t}=2.4 \mathrm{~T}$ was included. This is particularly important for the scattering of particles and for the opening angle of daughter particle showers.

Metals with a high atomic number usually have a high electron stopping power. Tungsten additionally has a very high melting temperature above $3400{ }^{\circ} \mathrm{C}$ and so it was chosen for the first tests. The total linear electron stopping power of tungsten is shown in Fig. 1(a) for the relevant electron energies. The other materials, which will be discussed, are included as well. The linear stopping power gives the energy loss of an electron which propagtes through $1 \mathrm{~cm}$ of the material. It is calculated as the product of the mass stopping power and the density of the material. The mass stopping power is taken from the ESTAR database. ${ }^{36}$ The total linear electron stopping power is given in Fig. 1(a). It is a sum of the collisional and the radiative contribution. The penetration depth of the REs in tungsten is only a few millimeters which leads to the melting of the material despite its high melting point. The radiative contribution to the total linear electron (a)

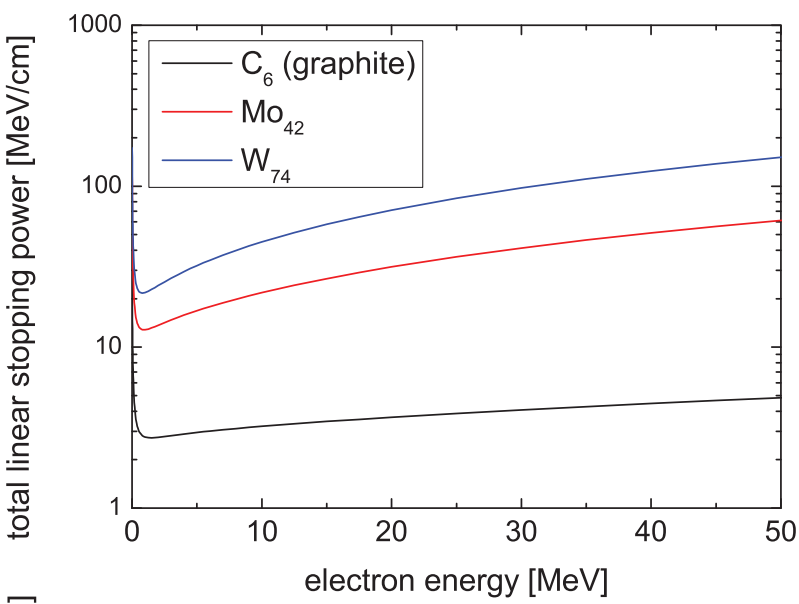

(b)

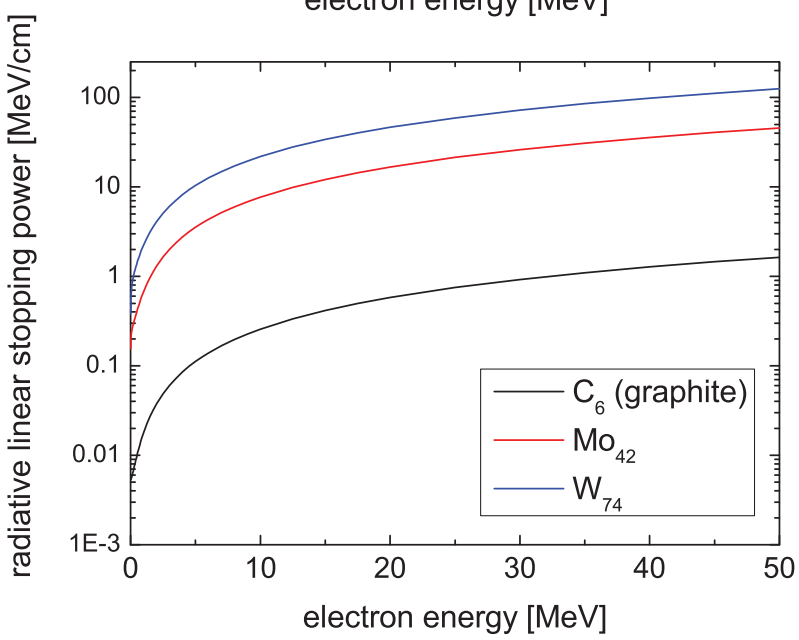

$(c)$

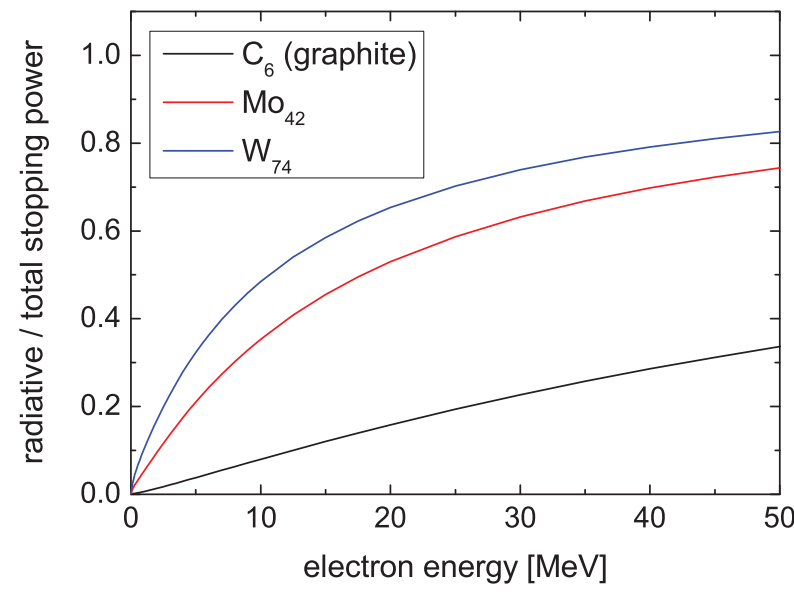

FIG. 1. Linear electron stopping powers of graphite, molybdenum and tungsten; calculated as the products of the densities and the mass stopping powers according to the ESTAR database. (a) Total linear electron stopping power including the radiative and collisional contributions. (b) Radiative linear electron stopping power. (c) Ratio of radiative to total electron stopping power.

stopping power of tungsten can be seen in Fig. 1(b). This contribution is relatively large as can be seen in Fig. 1(c). The ratio of the radiative to the total electron stopping power is shown. The generation of X-rays and $\gamma$-radiation is unfavorable because this radiation mainly leaves the material without heating it. Thus, it does not contribute to the measurement of the RE energy. Graphite has a low atomic number. The total and radiative linear electron stopping 


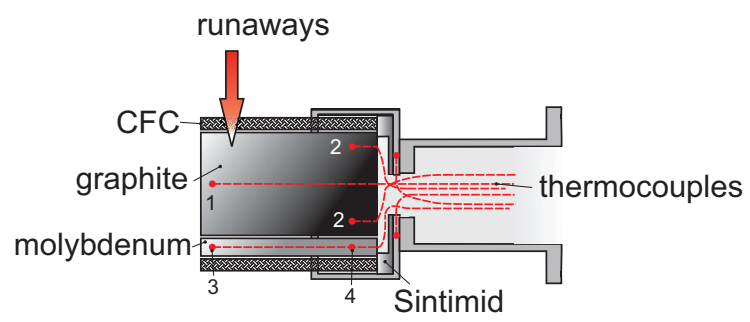

FIG. 2. Scheme of the calorimeter probe. A carbon fibre composite (CFC) is used as a shield against the plasma. The heating of an EK98 graphite and a molybdenum slab by the RE is measured by thermocouples in the material, which is thermally insulated against the probe holder by a slab of Sintimid.

powers are given in the Figs. 1(a) and 1(b), respectively. Both are substantially smaller than for tungsten. The sublimation temperature of graphite is above $3700{ }^{\circ} \mathrm{C}$. The REs easily propagate through several centimeters of graphite without causing a critical heating. Fig. 1(c) shows that the losses due to $\gamma$-radiation are substantially smaller than in tungsten. To stop the RE beam, a long slab of graphite would be optimal but the limitations on the probe's dimensions require a more elaborated design. After the REs have lost a major part of their energy in a slab of graphite, a material with a moderately high atomic number is needed to take the remaining RE energy. Molybdenum seems to be the optimal choice for this. It is nontoxic and it has a melting temperature above $2600{ }^{\circ} \mathrm{C}$. The total linear stopping power (Fig. $1(a))$ is high enough to stop the priorly decelerated REs. The test simulations predict no melting of the molybdenum. The radiative linear stopping power (Fig. 1(b)) is quite large compared to graphite. Fig. 1(c) shows the ratio of the radiative to the total stopping power for the relevant electron energies. As the REs are slowed down before they enter the molybdenum, the amount of $\gamma$-radiation is tolerable.

The probe design, which resulted from the test simulations, is shown in Fig. 2. The probe consists of a sandwich of materials in a stainless steel holder. Two $4 \mathrm{~mm}$ thick slabs of a carbon fibre composite (CFC) shield the inner probe parts against the thermal plasma in the directions of the electrons and ions which propagate along the magnetic field lines, respectively. The CFC conveniently takes the thermal loads and it does not break due to the occuring stresses. Thus, the CFC is much more suitable to shield the probe than fine grain graphite which tended to crack in earlier RE probes. The inner probe parts, which are designed to stop the REs, do not experience comparable thermal shocks and can be made of isotropic material. A $35 \mathrm{~mm}$ thick slab of EK98 fine grain graphite and a $6 \mathrm{~mm}$ thick slab of molybdenum are chosen as a result of the test simulations.

The heat exchange between the slabs and heat losses due to conduction are minimized by vacuum gaps between the $\mathrm{CFC}$, the isotropic graphite and the molybdenum. A slab of an amorphous high temperature polyimide, called Sintimid, is used to insulate the probe materials from the stainless steel holder. The coefficient of thermal conductivity of Sintimid is $\lambda=0.22 \mathrm{~W} /(\mathrm{K} \cdot \mathrm{m})$ at $23^{\circ} \mathrm{C}$ as specified by the manufacturer. The maximum working temperature of Sintimid is 350 ${ }^{\circ} \mathrm{C}$. Besides conduction, thermal radiation cools the probe between the discharges. The losses, which are due to radiation during the discharge and the following few tens of seconds of the temperature measurement, are minor: The radiation losses through the material surfaces, which face the plasma, are estimated in terms of black body radiation. At a temperature of $1000{ }^{\circ} \mathrm{C}$ the graphite surface radiates $80 \mathrm{~W}$ and the molybdenum surface $14 \mathrm{~W}$. At a temperature of $300{ }^{\circ} \mathrm{C}$ the graphite radiates $0.6 \mathrm{~W}$ through the front surface and the molybdenum radiates $0.1 \mathrm{~W}$. As the measured $\mathrm{RE}$ energies are in the $\mathrm{kJ}$ range, the losses due to thermal radiation can be neglected. In addition, the evaluation compensates for the losses due to conduction and radiation at the peak temperatures.

The thermocouples, which are used to measure the heating of the two sensitive slabs, are of the type K. Their measuring tips are positioned $5 \mathrm{~mm}$ below the front surface, which faces the plasma, and $5 \mathrm{~mm}$ away from the opposite surfaces of the slabs. In order to monitor the heating of the holder and the Sintimid, two thermocouples are placed in the Sintimid as well.

A $100 \mathrm{kA}$ RE beam, which is typical for a disruption with REs in TEXTOR, ${ }^{29}$ was simulated to fully strike the probe applying the Geant4 code. The spectral and radial distributions of the beam were chosen as described above for the test simulations. The calculations showed that about $20(30) \%$ of the REs with energies of 1(2) MeV can propagate through the CFC. This fraction increases to almost $60 \%$ for the REs which carry $3 \mathrm{MeV}$. The heating of the isotropic graphite and the molybdenum slab due to the impact of a RE beam is shown in Fig. 3. The vertical axes represent the direction in which the REs propagate into the materials. The horizontal axes give the direction of the radial decay of the RE beam. The colour codes show the heating of the materials due to the RE impact. The initial temperature profiles decrease strongly in the radial direction and with the material depth. The exponential radial decay of the RE beam causes the molybdenum to have its maximum of the temperature profile at the front surface as can be seen in Fig. 3(b). Fig. 3(a) shows that in the graphite this maximum of the temperature profile is not at the edge but a few $\mathrm{mm}$ radially inside. This is an edge layer effect which stems from the scattering of the REs in the CFC in front of the graphite. The effect varies if large changes of the magnetic field $B_{t}$ are applied. It is shown that the temperatures in the isotropic graphite and the molybdenum due to the impact of the REs from one disruption stay well below the critical values. $67.6 \%$ of the incident RE energy are deposited in the two sensitive slabs. The rest is lost via deposition in the CFC, the generation of $\gamma$-radiation, scattering out of the probe or via particles which leave the probe through the backside. Thus, a scaling factor of 1.48 has to be applied to the RE energies, which are obtained from the temperature rise, in order to estimate the total RE energy in the disruption. The scaling factor depends on the RE energy spectrum used in the simulations. The latter is chosen according to the latest measurements during TEXTOR disruptions. ${ }^{6,21}$

\section{OPERATIONAL SCENARIO}

The experiments were carried out at the tokamak TEXTOR which has a major radius of $1.75 \mathrm{~m}$. The minor radius 

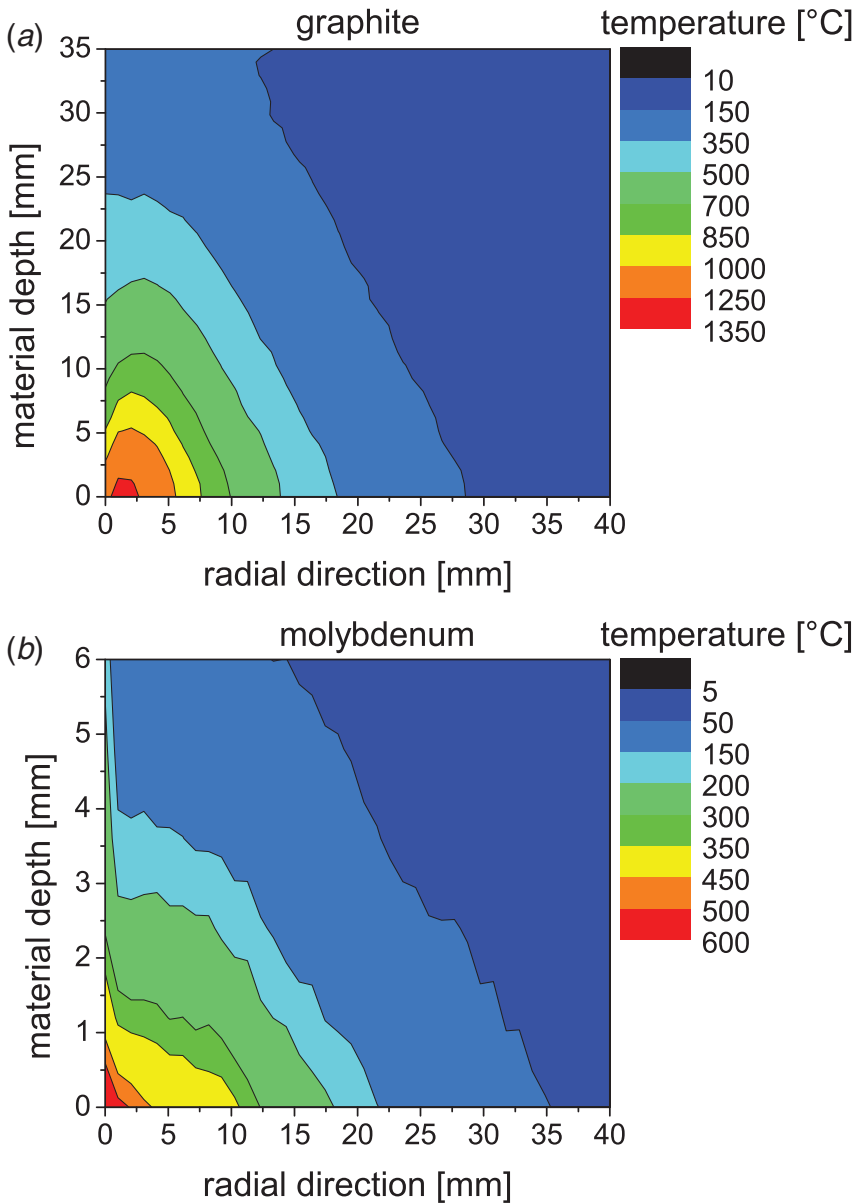

FIG. 3. Geant4 code simulation of the impact of a RE beam from one TEXTOR disruption onto the calorimeter probe. (a) Temperature profile of the EK98 graphite part after the RE impact. (b) Temperature profile of the molybdenum part after the RE impact.

of $0.46 \mathrm{~m}$ is defined by the ALT-II - a toroidal belt limiter, which is positioned $45^{\circ}$ below the midplane at the low field side. The disruptions were induced by the injection of 0.05 bar 1 of argon gas into ohmic discharges. Prior to the gas injection, the electron density was $2 \cdot 10^{19} \mathrm{~m}^{-3}$ throughout the campaign. The valve was triggered $2 \mathrm{~s}$ after the startup $^{24,37}$ of the discharge. The typical temporal evolution of the loop voltage and the plasma current during a disruption of the campaign can be seen in the Figs. 4(a) and 4(b), respectively. The main parameters of this particular discharge are the toroidal magnetic field of $B_{t}=2.4 \mathrm{~T}$ and the predisruptive plasma current of about $I_{p}=250 \mathrm{kA}$. The thermal quench of the disruption starts with the negative spike in the loop voltage at about $2.005 \mathrm{~s}$. The following steep increase of the loop voltage enables the acceleration of the plasma electrons into the runaway regime. The almost collisionless REs form a plateau in the plasma current and thus retard the current quench as can be seen in Fig. 4(b). The figure also shows an exponentially decaying fit which can be subtracted from the plasma current to calculate the maximum $\mathrm{RE}$ current. The injection of argon reliably triggers the generation of REs during the current quench of TEXTOR disruptions. ${ }^{29}$ The generation of the RE population was checked by the detection of the suprathermal electron cyclotron emission and neutrons. REs with energies above $10 \mathrm{MeV}$ produce

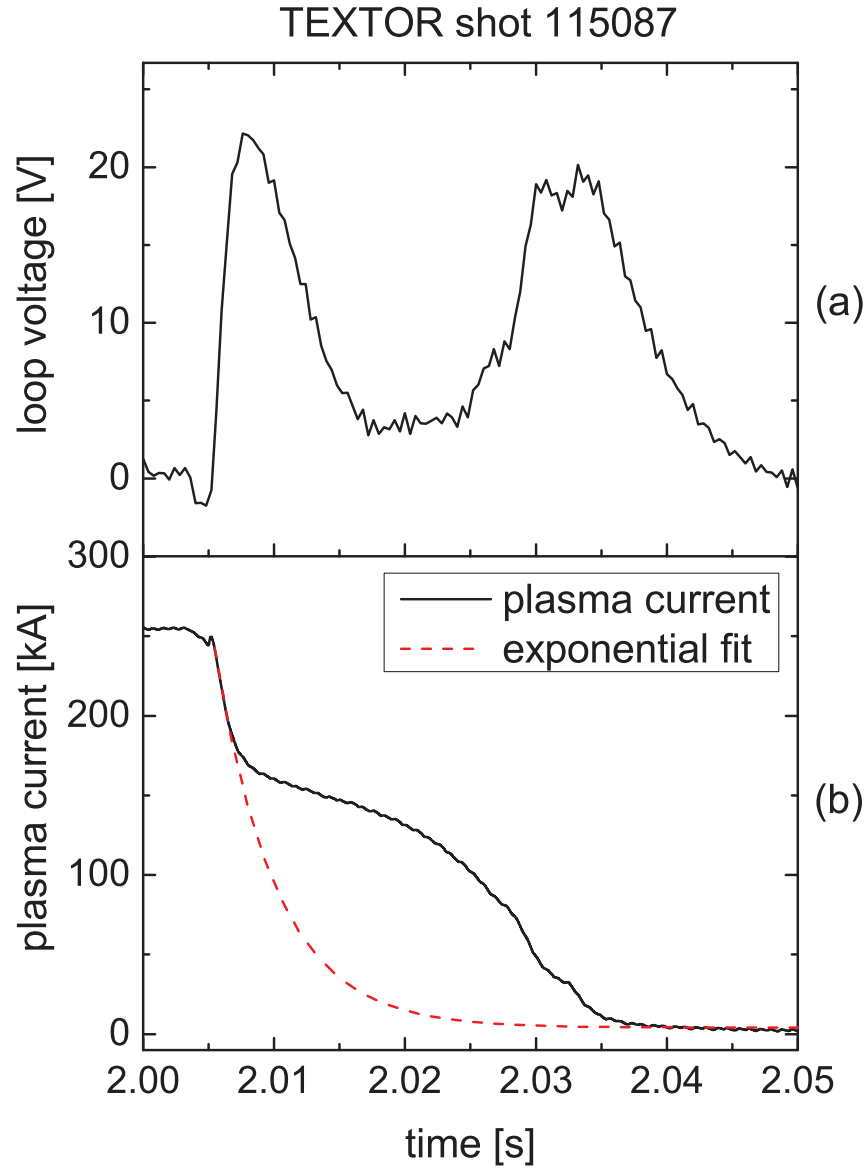

FIG. 4. Temporal evolution of (a) the loop voltage and (b) the plasma current during a typical disruption of the campaign.

neutrons by photonuclear reactions with the graphite of the PFCs. ${ }^{11,14}$ The vertical field coils of TEXTOR were used to direct the RE beam via feed forward to the low field side of the machine where the probe was inserted radially in the equatorial midplane. The geometry of the setup is shown as a scheme of a poloidal cross section in Fig. 5. The radius of the RE beam is assumed to be considerably smaller than the radius of the plasma column prior to the disruption. This assumption is justified by the statistical variations of the RE probe measurements as it is shown in Sec. IV. About $50 \mathrm{~ms}$ prior to the gas injection, the probe was inserted to its measuremental position where it remained for about $200 \mathrm{~ms}$. The radial position of the probe, $B_{t}$ and the plasma current were separately scanned from shot to shot.

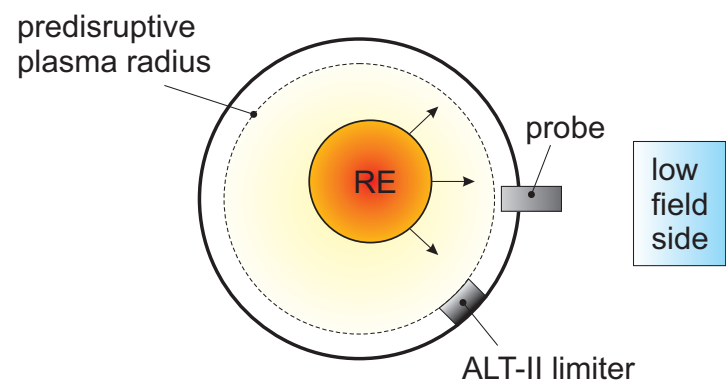

FIG. 5. Scheme of the poloidal cross section of TEXTOR showing the geometry during the probe measurement. 
During the disruption, shown in Fig. 4, the calorimeter probe was inserted to a minor radius of $r_{\text {cal }}=46 \mathrm{~cm}$. The heating of the isotropic graphite slab due to the RE impact can be seen in Fig. 6(a). The steeply rising temperature curve was measured by the tip of a thermocouple $5 \mathrm{~mm}$ below the front surface which faces the plasma. The thermocouples at this position are labeled by 1 in Fig. 2. The two slowly rising temperature curves in Fig. 6(a) belong to thermocouples radially more outside. They are labeled by 2 in Fig. 2 . The different curves join after about $60 \mathrm{~s}$ at the equilibrium temperature which then can be assumed for the whole slab. Similar results were obtained for the molybdenum slab. Here the equilibrium temperature is reached after about $20 \mathrm{~s}$ as shown in Fig. 6(b). The dashed lines of best fit in the Figs. 6(a) and 6(b) are used to determine the equilibrium temperature. This procedure compensates for errors due to heat losses via thermal radiation and heat conduction. In addition, the heat losses are minimized due to the probe's thermal insulation. A side effect of the thermal insulation is a shot to shot increase of the probe temperature. This progressive heating of the probe does not influence the energy deposition of the
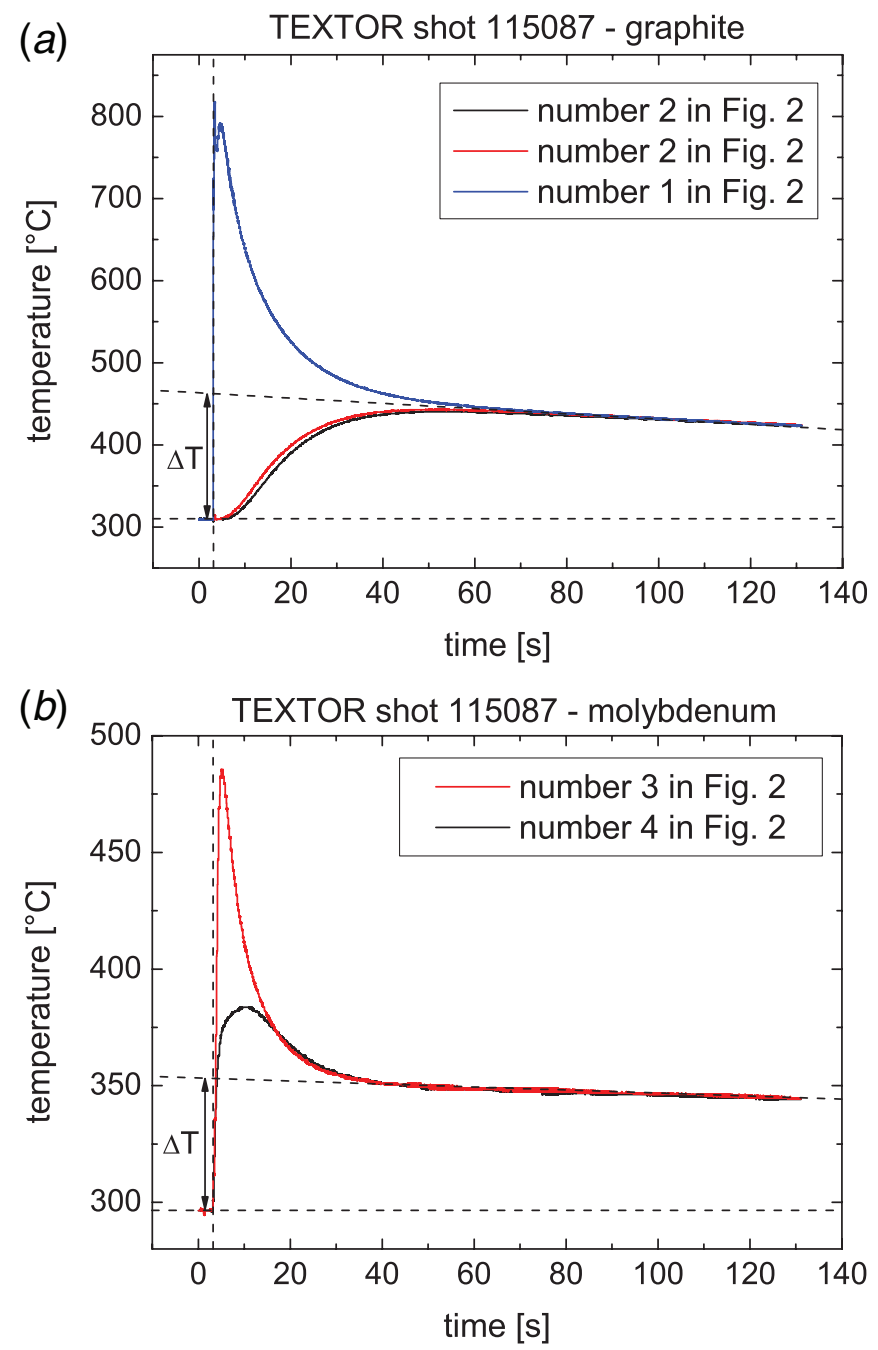

FIG. 6. Typical calorimetric measurement during a RE impact with added dashed lines used for the estimation of the temperature increase. (a) Signals of thermocouples inside the EK 98 graphite. (b) Signals of thermocouples inside the molybdenum.

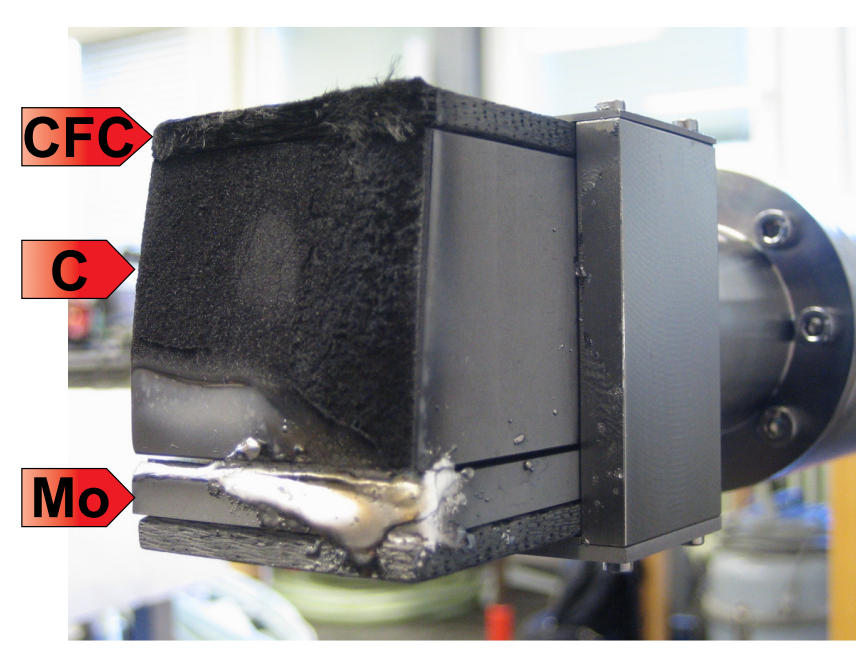

FIG. 7. Photograph of the calorimeter probe taken after the experiments, with three arrows indicating the erosion of the CFC, the isotropic graphite (C) and the melting of the molybdenum (Mo), respectively.

REs in the materials. Thus, the temperature differences, which are caused by the RE impacts and which determine the estimation of the RE energies, are independent of the absolute probe temperature. The probe materials just have to be kept from reaching their melting temperatures by cooldown phases between the discharges. Fig. 7 shows a photograph of the probe head. It was taken after the experimental campaign. The isotropic graphite and the $\mathrm{CFC}$, which faced the impinging electrons, are eroded and the molybdenum is partly molten due to the progressive heating of the probe. These damages are the cause for a difference between the geometrically calculated mass of the materials and the mass measured after the campaign. This mass difference accounts to about $5 \%$ for the isotropic graphite and to below $1 \%$ for the molybdenum. The significance of these uncertainties can be seen in terms of the error bars in Fig. 8(a).

Using the measured temperature differences and the scaling factor, which was calculated in Sec. II, the RE energies in the disruptions are estimated.

\section{RUNAWAY ENERGY}

The experimental results include the RE currents and the RE energies in the disruptions. The RE currents are calculated by subtracting exponentially decaying fits from the $\mathrm{RE}$ plateaus. The RE energies are measured by the calorimeter probe. Additional results are used as an independent check for the calorimetry data: A scintillator probe measured RE energy spectra at the plasma edge during TEXTOR disruptions. ${ }^{6}$ These disruptions were induced in the same way as described in Sec. III. The total numbers of the REs in the disruptions were estimated from the RE currents. The energy spectra were scaled up to contain this number of REs.

There are two ways to evaluate the RE currents and energies. Both should be discussed. The RE currents show large fluctuations from shot to shot as the generation of the REs is sensitive to the exact evolution of the disruption. Most natural disruptions do not show REs at all. At TEXTOR the induced disruptions are used for systematical studies of the physics of the REs. Thus, they favor the generation 

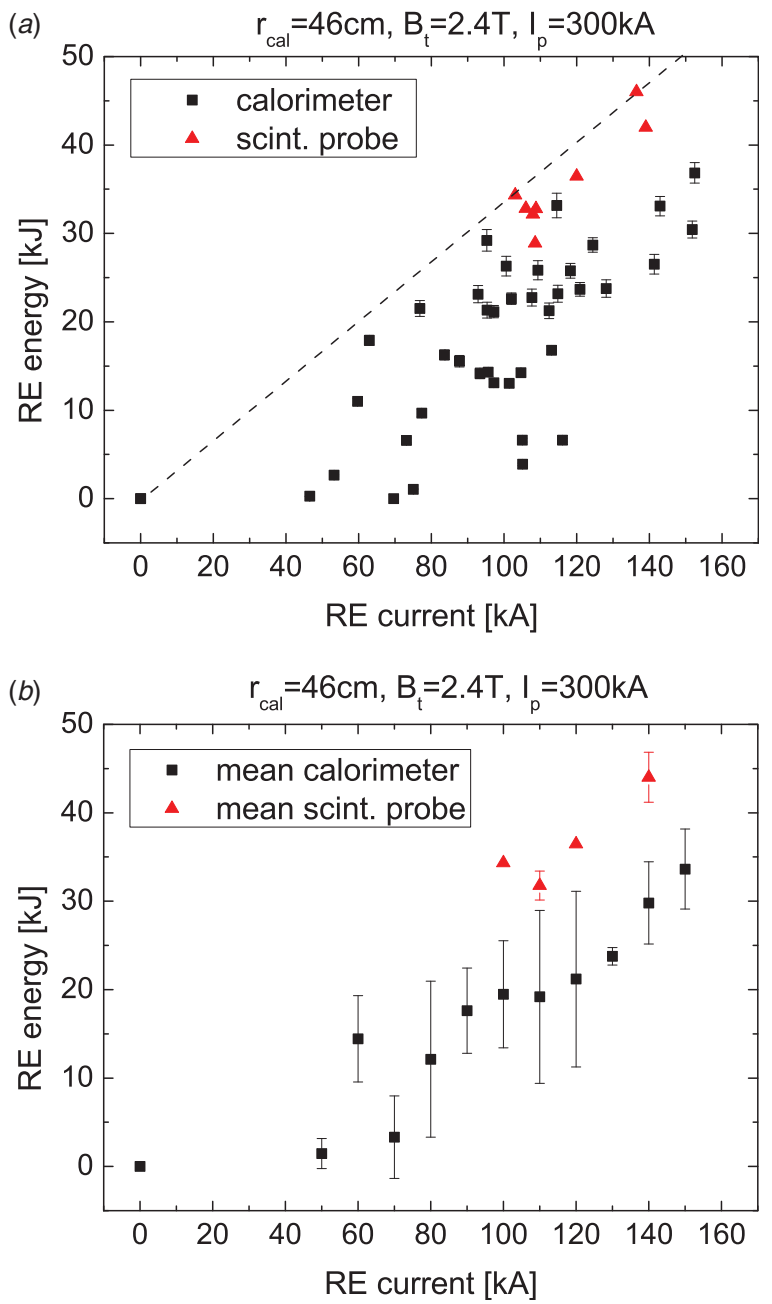

FIG. 8. RE energies, measured by the calorimeter probe and resulting from scintillator probe studies, over the RE current. (a) Overview of the measured values and a dashed line which indicates an upper limit according to the geometrical interpretation of the data. (b) Mean values of the RE energy.

of REs. Generalizing the results, the RE currents should be seen as maximum values. In the following the evaluation according to this interpretation (a) is based on the upper limits of the achieved RE currents. The interpretation (b) applies a statistical standard method. The scatter of the data is reduced by taking the mean of the RE currents for fixed values of the plasma parameters. The RE energies allow an analogue treatment. The large scatter of the calorimetry data points is caused by the RE beam often striking the probe only partially or even missing it. This implies a RE beam, which is smaller than the predisruptive plasma column. This assumption is in agreement with the increase of the internal inductance in postdisruptive RE plasmas. ${ }^{38}$ The statistical variations cause the calorimeter measurements to underestimate the RE energy. If the RE beams were fully incident on the probe, they produced data points at an upper limit. The scintillator probe results are independent of this geometrical interpretation (a). Thus, they are less scattered and they are close to the upper limit. In analogy to the RE currents the evaluation (b) reduces the data scatter by taking the mean of the RE energies. This statistical evaluation ignores the geometrical interpretation and leads to different absolute values. (a)

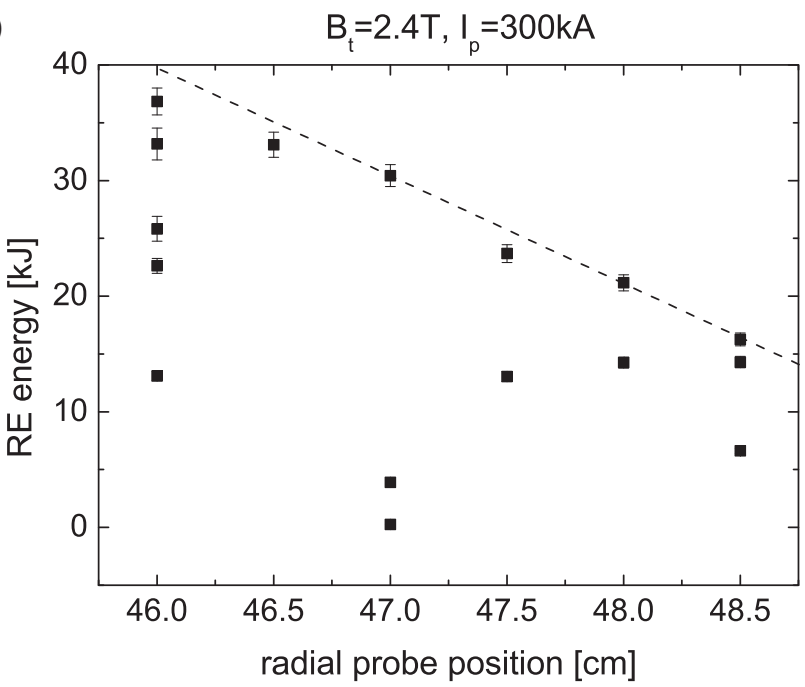

(b)

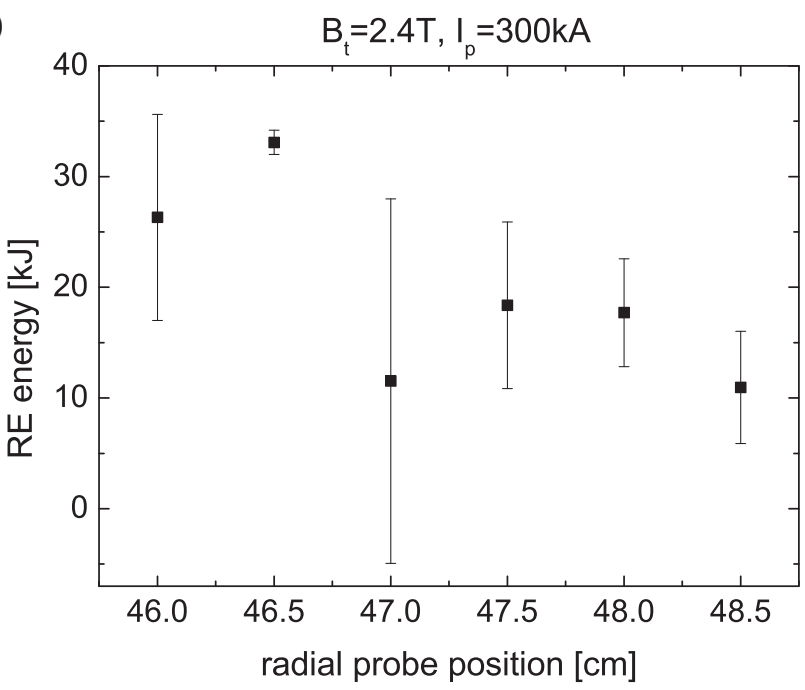

FIG. 9. RE energies over the radial position of the calorimeter probe. (a) Overview of the measured values and a dashed line which indicates an upper limit according to the geometrical interpretation of the data. (b) Mean RE energies.

Fig. 8 shows the RE energies which were measured by the calorimeter probe at the radial position $r_{c a l}=46 \mathrm{~cm}$ during disruptions with $B_{t}=2.4 \mathrm{~T}$ and $I_{p}=300 \mathrm{kA}$. The RE energies are shown in dependence of the RE currents. The scintillator results are also included. Fig. 8(a) shows the results according to the geometrical interpretation. The upper limit of the RE energies is indicated by the dashed line. The scintillator probe results are close to this line and support the geometrical interpretation. The mean RE energies according to the statistical interpretation can be seen in Fig. 8(b). The relative dependence of the RE energy on the RE current is clearer in this figure. The scintillator results are independent of the geometrical fluctuations and thus their mean values are larger than the mean calorimetry data. Both ways of evaluating the data suggest an approximately linear increase of the RE energy for RE currents above $50 \mathrm{kA}$. The mean RE current of all the disruptions in Fig. 8(a) is about $100 \mathrm{kA}$. According to the geometrical interpretation, a typical TEXTOR disruption includes a RE energy content of 30 to $35 \mathrm{~kJ}$. The mean RE energy of all the disruptions is $20 \pm 12 \mathrm{~kJ}$. These results have been extrapolated to RE currents of 
1 MA, typical of disruptions on JET. This allows a comparison with the results presented in the literature. ${ }^{34}$ The RE energy content of JET disruptions was inferred from the temperature increase of hot spots. This was done for 17 disruptions and the RE energy was found to vary between 0 and $0.5 \mathrm{MJ}$. The results show an accumulation of 4 data points around $0.32 \mathrm{MJ}$ in Fig. 4 of the reference. The according RE currents are not given in the publication. Rather a typical RE current of $1 \mathrm{MA}$ is stated to contain a RE energy of about 0.5 MJ. The extrapolation of the dashed line in Fig. 8(a) to a RE current of 1 MA leads to a RE energy of approximately 0.34 MJ. A linear fit of the calorimeter results in Fig. 8(b) leads to about $0.21 \mathrm{MJ}$ of RE energy in a current of $1 \mathrm{MA}$. Consequently, the comparison supports the geometrical interpretation of the TEXTOR results.

The dependence of the RE energy on the radial position of the calorimeter probe is presented in Fig. 9 for $B_{t}=2.4 \mathrm{~T}$ and $I_{p}=300 \mathrm{kA}$. The geometrical interpretation is shown in Fig. 9(a). A dashed line is included to mark the upper limit of the RE energy. Fig. 9(b) shows the mean values of the $\mathrm{RE}$ energy at each radial position. The minimum radius of

(a)
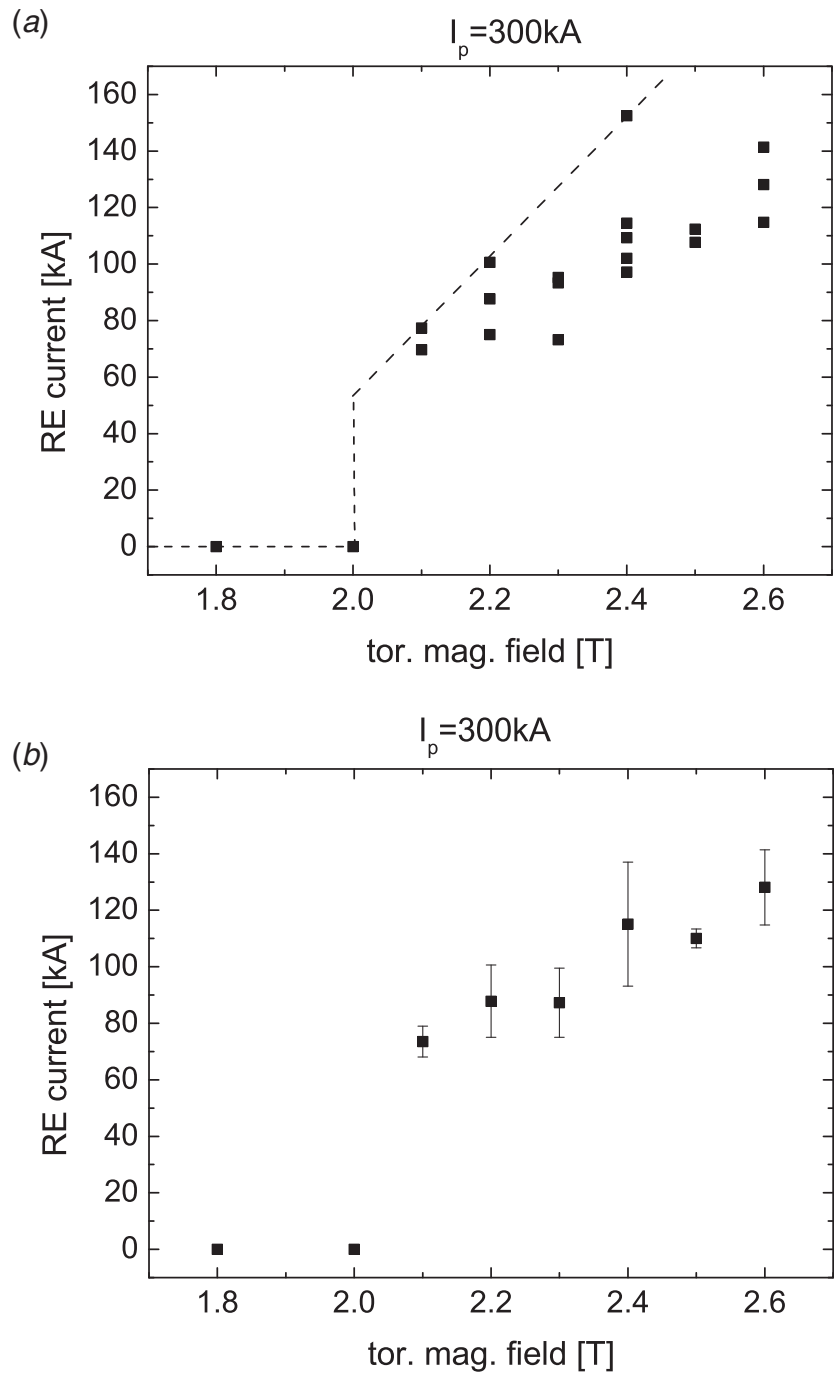

FIG. 10. RE currents over the toroidal magnetic field. (a) Overview of the measured RE currents and a dashed line which outlines the maximum values. (b) Mean RE currents. $r_{c a l}=46 \mathrm{~cm}$ corresponds to the position of the toroidal limiter. For probe positions radially more outside than $r_{c a l}=46.5 \mathrm{~cm}$ the energy decreases 9(a) linearly or 9(b) less than linearly. Thus, the fraction of the RE beam, which is stopped by the probe, decreases on the average. Consequently, the probe measurements are most reliable for $r_{c a l}=46.5 \mathrm{~cm}$. Going $2.5 \mathrm{~cm}$ away from the plasma edge, reduces the RE energy load on the PFCs by a factor of 2 .

The dependence of the RE current on the toroidal magnetic field is shown in Fig. 10 for $I_{p}=300 \mathrm{kA}$. Fig. 10(a) shows all the RE currents. The maximum values are outlined by a dashed line. The mean RE currents are given in Fig. 10(b). From both figures it can be concluded that for $B_{t} \leq 2 \mathrm{~T}$ no REs are produced. Such a RE generation threshold around $B_{t} \approx 2 \mathrm{~T}$ was observed on several machines. The existence of such a threshold was statistically analysed on JET (Ref. 39) and a comparison between JET and TEXTOR was made. ${ }^{40}$ Two physical mechanisms, which possibly could cause the threshold, were studied theoretically. ${ }^{41}$ "The first possible explanation $[\ldots]$ is that the runaway beam excites whistler

(a)
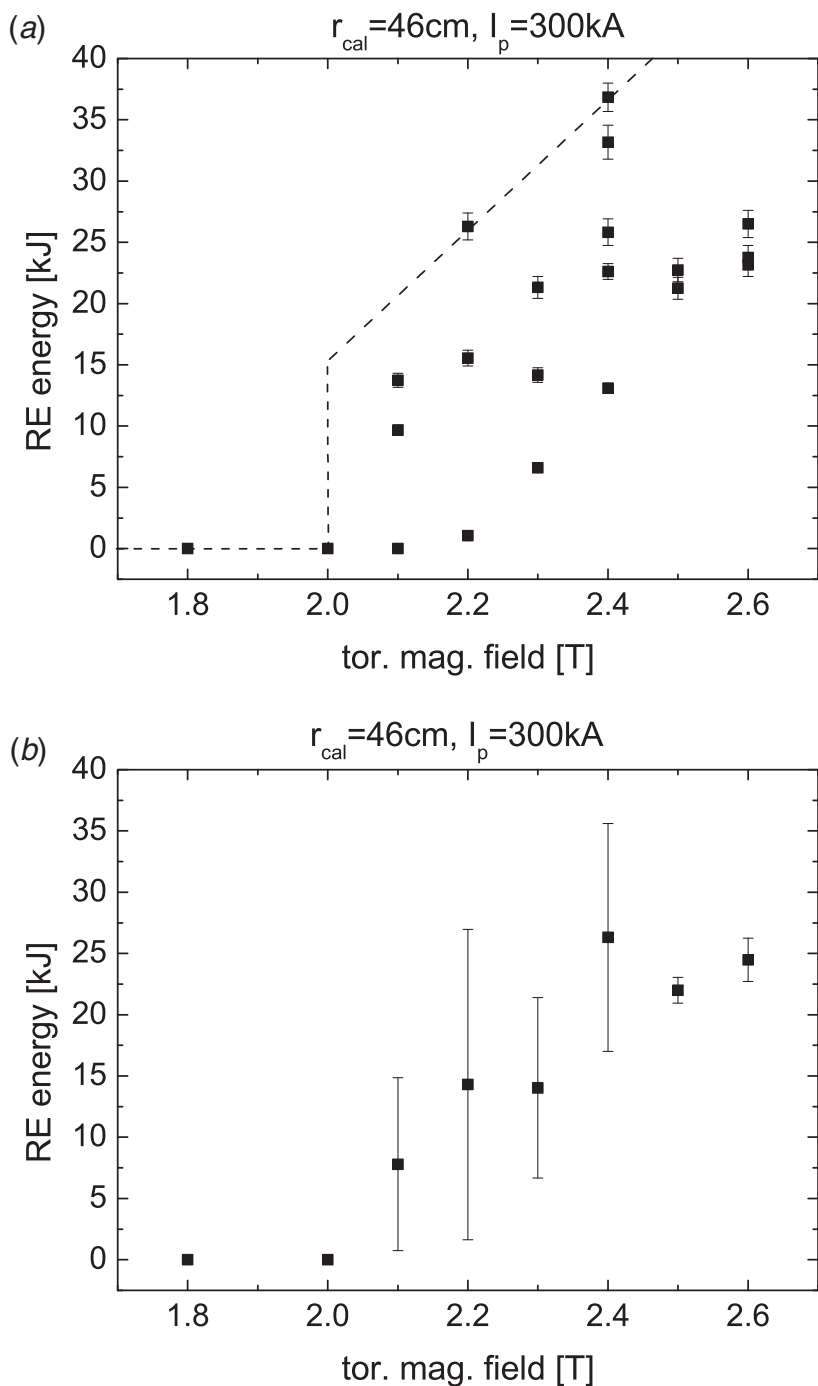

FIG. 11. RE energies, measured by the calorimeter probe, over the toroidal magnetic field. (a) Overview of the measured values and a dashed line which indicates an upper limit according to the geometrical interpretation of the data. (b) Mean RE energies. 
waves that scatter the electrons in velocity space $[\ldots]$ The second possible explanation is the magnetic field dependence of the criterion for substantial runaway production obtained by calculating how many runaway electrons can be produced before the induced toroidal electric field diffuses out of the plasma" (abstract). Above the threshold the RE current increases approximately linearly with $B_{t}$. The slope of the increase is higher in Fig. 10(a) than in Fig. 10(b). The RE energies measured for $I_{p}=300 \mathrm{kA}$ at $r_{c a l}=46 \mathrm{~cm}$ are presented over $B_{t}$ in Fig. 11. The geometrical interpretation including an upper limit is given in Fig. 11(a) and the mean RE energies are given in Fig. 11(b). A similar $B_{t}$-dependence as for the RE current applies to the RE energy. This supports the conclusion of a linear dependence of the RE energy on the RE current. Again the slope in Fig. 11(a) is steeper than in Fig. 11(b).

The dependence of the RE current on the predisruptive plasma current can be seen in Fig. 12 for $B_{t}=2.4 \mathrm{~T}$. Again all the RE currents and an upper limit are given in Fig. 12(a) and the mean RE currents are given in Fig. 12(b). According to both figures, the RE current increases for

(a)

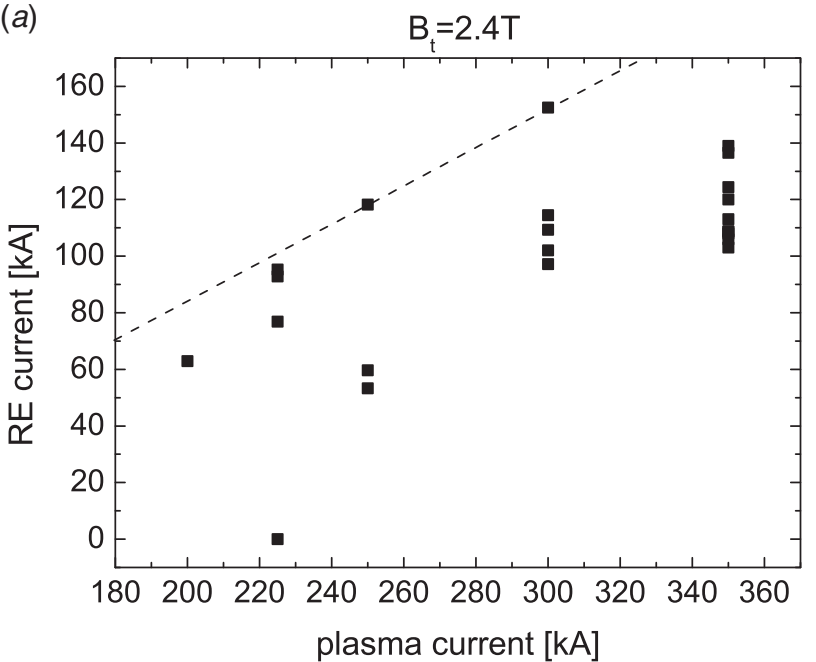

(b)

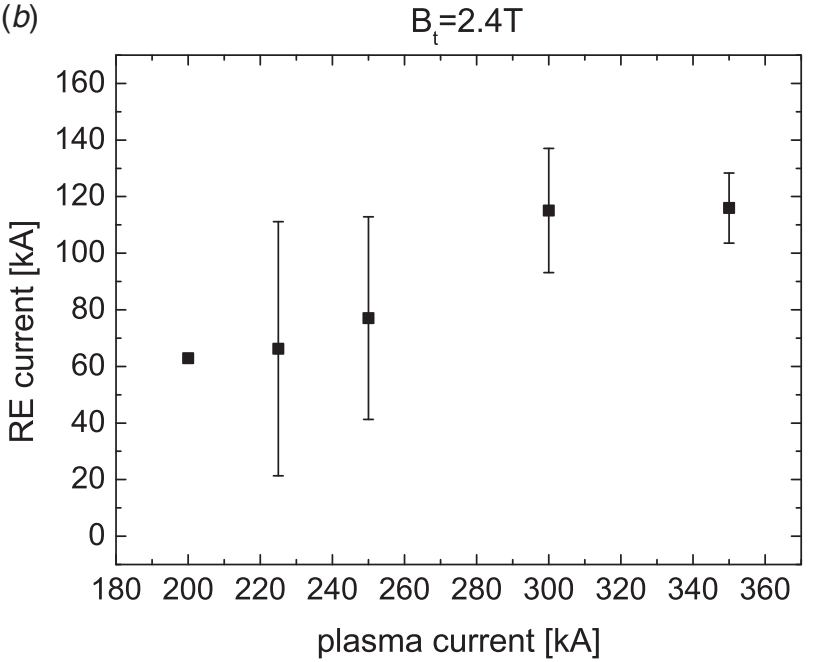

FIG. 12. RE currents over the predisruptive plasma current. (a) Overview of the measured RE currents and a dashed line which outlines the maximum values. (b) Mean RE currents.
$200 \mathrm{kA} \leq I_{p} \leq 300 \mathrm{kA}$. According to Fig. 12(b) the RE current seems not to increase above $I_{p}=300 \mathrm{kA}$. Fig. 13 shows the dependence of the RE energy on the predisruptive plasma current. The calorimeter probe measured at $r_{\text {cal }}=46 \mathrm{~cm}$. The scintillator results are included as well. Fig. 13(a) gives the upper limit on the RE energy which results from the geometrical interpretation. Fig. 13(b) shows the mean RE energies. Both figures allow a similar estimate of the $I_{p}$-dependence of the RE energy as for the RE current in Fig. 12. Taking all the disruptions into account, which resulted in a nonzero RE current, the mean conversion of the predisruptive plasma current into a RE current is found to be $35 \%$. The maximum value is $51 \%$.

The efficiency of the conversion of predisruptive magnetic plasma energy into RE energy is calculated. For this, the magnetic energy of the plasma prior to the disruption is calculated via $E_{\text {mag }}=\frac{1}{2} I_{p}^{2} L_{p}$. The inductance is $L_{p}=\mu_{0} R\left(\ln (8 R / a)-2+\ell_{i} / 2\right)$. The value of the internal inductance is the only parameter which is not exactly known
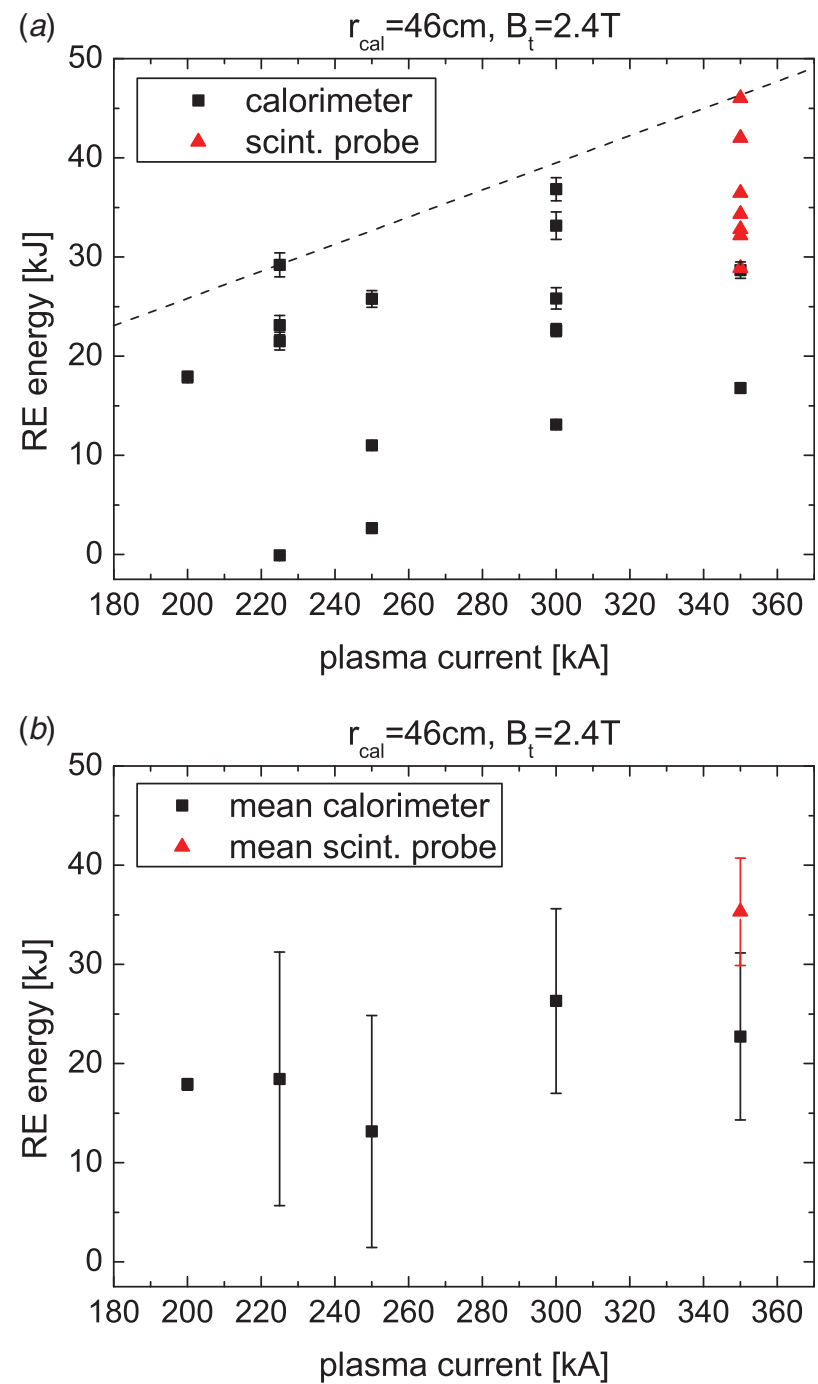

FIG. 13. RE energies, measured by the calorimeter probe and estimated from energy spectra measured by the scintillator probe, over the predisruptive plasma current. (a) Overview of the measured values and a dashed line which indicates an upper limit according to the geometrical interpretation of the data. (b) Mean RE energies. 

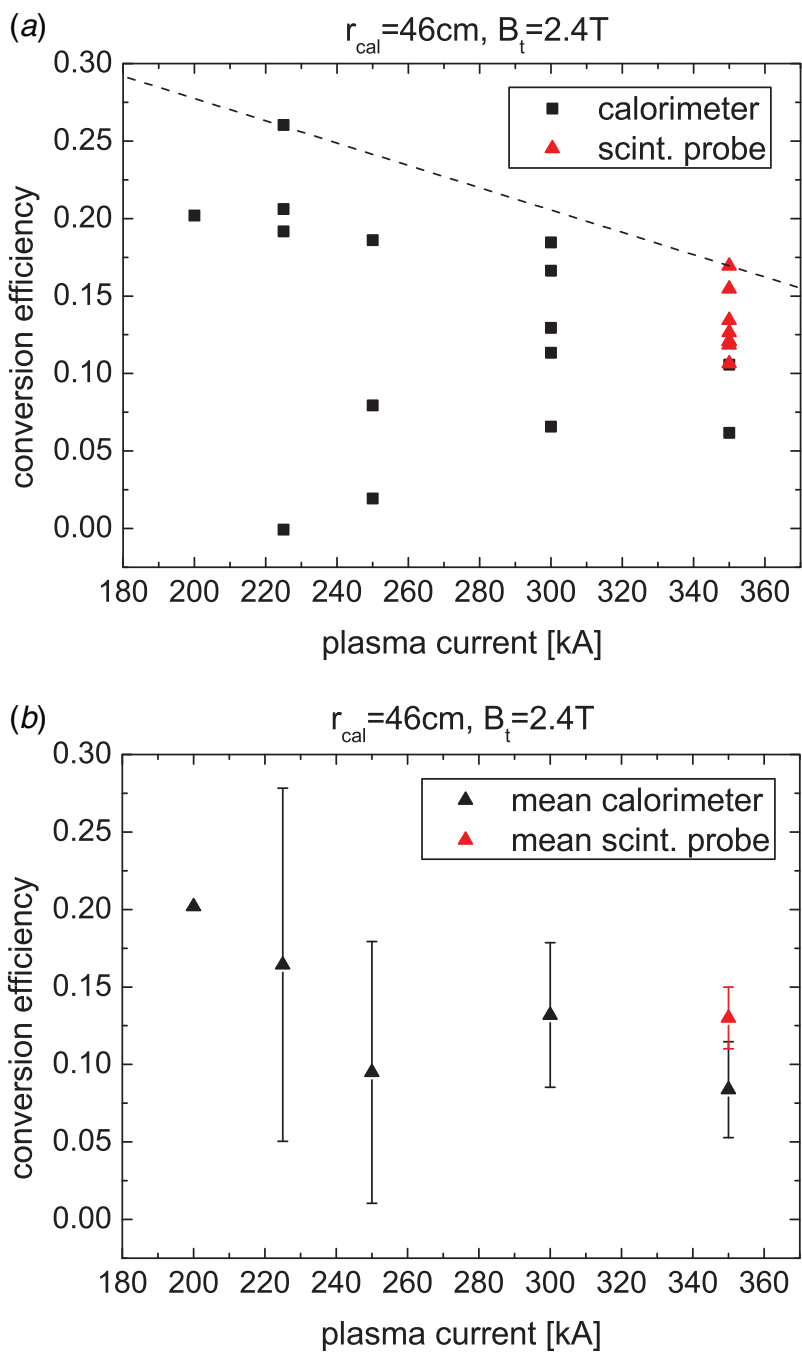

FIG. 14. Conversion efficiencies of the predisruptive magnetic plasma energy into RE energy over the plasma current for calorimeter and scintillator probe data. (a) Conversion efficiencies resulting from the measured RE energies and a dashed line which outlines the maximum values. (b) conversion efficiencies resulting from the mean RE energies.

for the predisruptive plasma but it is typically $\ell_{i}=1.2$ at TEXTOR. Deviations from this assumption by \pm 1 introduce an error of $\pm 3 \%$ to the final result. Fig. 12 shows the tendency of the conversion efficiency to decrease with increasing plasma current for $B_{t}=2.4 \mathrm{~T}$. The results according to the geometrical interpretation of the calorimeter probe measurements are given in Fig. 14(a). Again the results of the scintillator probe studies add very well to the new measurements. The maximum energy conversion is found to be $26 \%$. The conversion efficiencies which result from the mean values of the RE energy are given in Fig. 14(b). The $I_{p}$-dependence is very similar in both figures although the absolute values are smaller in Fig. 14(b).

\section{SUMMARY}

A calorimeter probe allowed direct measurements of the total RE energy in tokamak disruptions. A typical RE plateau current of $100 \mathrm{kA}$ was found to contain 30 to $35 \mathrm{~kJ}$ of RE energy at TEXTOR. The scaling of the RE energy with the $\mathrm{RE}$ current and thus the number of generated REs is approxi- mately linear for RE currents above $50 \mathrm{kA}$. The RE energy, which is deposited in the probe, decreases for radial probe positions more than $1.5 \mathrm{~cm}$ behind the limiter. The RE energy load onto PFCs is reduced by a factor of 2 by moving them $2.5 \mathrm{~cm}$ radially away from the plasma. The RE current and the RE energy show a RE generation threshold at $B_{t} \approx 2 \mathrm{~T}$. Above the threshold the $\mathrm{RE}$ current increases approximately linearly with $B_{t}$. This dependence is reproduced for the RE energy. The variation of the RE current with the plasma current could also be reproduced for the RE energy, which strengthens the conclusion of a linear dependence of the RE energy on the RE current. The conversion of the predisruptive, magnetic plasma energy into RE energy during the current quench shows a tendency to decrease with the plasma current. The maximum of the conversion was found at $26 \%$. Thus, the work presented provides additional insight on the RE energy conversion during tokamak disruptions. These results should be taken into account in future simulations of REs.

\section{ACKNOWLEDGMENTS}

The authors would like to thank Jochen Linke and Corinna Thomser for the fruitful discussions.

This work was funded by the GRK 1203, an F and E contract and the Trilateral Euregio Cluster (TEC).

${ }^{1}$ Plasma Control ITER Physics Expert Group on Disruptions, MHD, and ITER Physics Basis Editors, Nucl. Fusion 39, 2251 (1999).

${ }^{2}$ V. V. Plyusnin, V. Riccardo, R. Jaspers, B. Alper, V. G. Kiptily, J. Mlynar, S. Popovichev, E. de La Luna, F. Andersson, and JET EFDA contributors, Nucl. Fusion 46, 277-284 (2006).

${ }^{3}$ S. Putvinski, P. Barabaschi, N. Fujisawa, N. Putvinskaya, M. N. Rosenbluth, and J. Wesley, Plasma Phys. Controlled Fusion 39, B157-B171 (1997).

${ }^{4}$ P. Helander, L.-G. Eriksson, and F. Andersson, Plasma Phys. Controlled Fusion 44, B247-B262 (2002).

${ }^{5}$ R. D. Gill, Nucl. Fusion 33, 1613-1625 (1993).

${ }^{6}$ M. Forster, K. H. Finken, T. Kudyakov, M. Lehnen, O. Willi, Y. Xu, and TEXTOR team, "Temporal and spectral evolution of runaway electron bursts in TEXTOR disruptions," Phys. Rev. Lett. (submitted).

${ }^{7}$ H.-W. Bartels, Fusion Eng. Des. 23, 323-328 (1993).

${ }^{8}$ R. Nygren, T. Lutz, D. Walsh, G. Martin, M. Chatelier, T. Loarer, and D. Guilhem, J. Nucl. Mater. 241-243, 522-527 (1997).

${ }^{9}$ M. Shimada et al., Nucl. Fusion 47, S1 (2007).

${ }^{10}$ T. C. Hender et al., Nucl. Fusion 47, S128 (2007).

${ }^{11}$ B. Esposito, J. R. Martín-Solís, F. M. Poli, J. A. Mier, R. Sánchez, and L. Panaccione, Phys. Plasmas 10, 2350/11 (2003).

${ }^{12}$ B. Esposito, R. M. Solis, P. van Belle, O. N. Jarvis, F. B. Marcus, G. Sadler, R. Sanchez, B. Fischer, P. Froissard, J. M. Adams, E. Cecil, and N. Watkins, Plasma Phys. Controlled Fusion 38, 2035-2049 (1996).

${ }^{13}$ P. V. Savrukhin, Phys. Rev. Lett. 86, 3036(4) (2001).

${ }^{14}$ T. Kawamura, H. Obayashi, and A. Miyahara, Fusion Eng. Des. 9, 45-48 (1989).

${ }^{15}$ K. H. Finken, B. Unterberg, Y. Xu, S. S. Abdullaev, M. Jakubowski, M. Lehnen, M. F. M. de Bock, S. Bozhenkov, S. Brezinsek, C. Busch, I. G. J. Classen, J. W. Coenen, D. Harting, M. von Hellermann, S. Jachmich, R. J. E. Jaspers, Y. Kikuchi, A. Krämer-Flecken, Y. Liang, M. Mitri, P. Peleman, A. Pospieszczyk, D. Reiser, D. Reiter, U. Samm, D. Schega, O. Schmitz, S. Soldatov, M. Van Schoor, M. Vergote, R. R. Weynants, R. Wolf, O. Zimmermann, and the TEXTOR team, Nucl. Fusion 47, 522-534 (2007).

${ }^{16}$ K. H. Finken, S. S. Abdullaev, M. W. Jakubowski, R. Jaspers, M. Lehnen, R. Schlickeiser, K. H. Spatschek, A. Wingen, R. Wolf, and the TEXTOR team, Nucl. Fusion 47, 91-102 (2007).

${ }^{17}$ R. Jaspers, N. J. Lopes Cardozo, A. J. H. Donn, H. L. M. Widdershoven, and K. H. Finken, Rev. Sci. Instrum. 72, 466 (2001).

${ }^{18}$ K. H. Finken, J. G. Watkins, D. Rusbuldt, W. J. Corbett, K. H. Dippel, D. M. Goebel, and R. A. Moyer, Nucl. Fusion 30, 859-870 (1990). 
${ }^{19}$ R. Jaspers, N. J. Lopes Cardozo, F. C. Schller, K. H. Finken, T. Grewe, and G. Mank, Nucl. Fusion 36, 367-373 (1996).

${ }^{20}$ T. Kudyakov, K. H. Finken, M. W. Jakubowski, M. Lehnen, Y. Xu, B. Schweer, T. Toncian, G. van Wassenhove, O. Willi, and the TEXTOR team, Nucl. Fusion 48, 122002 (2008).

${ }^{21}$ M. Forster, K. H. Finken, M. Lehnen, J. Linke, B. Schweer, C. Thomser, O. Willi, Y. Xu, and the TEXTOR team, Nucl. Fusion 51, 043003 (8pp) (2011).

${ }^{22}$ T. Kudyakov, K. H. Finken, M. Jakubowski, M. Lehnen, Y. Xu, and O. Willi, Rev. Sci. Instrum. 79, 10F126 (2008).

${ }^{23}$ D. G. Whyte, T. C. Jernigan, D. A. Humphreys, A. W. Hyatt, C. J. Lasnier, P. B. Parks, T. E. Evans, M. N. Rosenbluth, P. L. Taylor, A. G. Kellman, D. S. Gray, E. M. Hollmann, and S. K. Combs, Phys. Rev. Lett. 89, 055001 (2002).

${ }^{24}$ S. A. Bozhenkov, M. Lehnen, K. H. Finken, M. W. Jakubowski, R. C. Wolf, R. Jaspers, M. Kantor, O. V. Marchuk, E. Uzgel, G. VanWassenhove, O. Zimmermann, D. Reiter, and the TEXTOR team, Plasma Phys. Controlled Fusion 50, 105007 (2008).

${ }^{25}$ S. A. Bozhenkov, M. Lehnen, K. H. Finken, G. Bertschinger, H. R. Koslowski, D. Reiter, R. C. Wolf, and the TEXTOR team, Nucl. Fusion 51, 083033 (2011).

${ }^{26}$ G. Pautasso, D. Coster, T. Eich, J. C. Fuchs, O. Gruber, A. Gude, A. Herrmann, V. Igochine, C. Konz, B. Kurzan, K. Lackner, T. Lunt, M. Marascheck, A. Mlynek, B. Reiter, V. Rohde, Y. Zhang, X. Bonnin, M. Beck, G. Prausner, and the ASDEX Upgrade Team, Plasma Phys. Controlled Fusion 51, 124056 (2009).

${ }^{27}$ S. C. Jardin, G. L. Schmidt, E. D. Fredrickson, K. W. Hill, J. Hyun, B. J. Merrill, and R. Sayer, Nucl. Fusion 40, 923-933 (2000).

${ }^{28}$ N. Commaux, L. R. Baylor, T. C. Jernigan, E. M. Hollmann, P. B. Parks, D. A. Humphreys, J. C.Wesley, and J. H. Yu, Nucl. Fusion 50, 112001 (2010).

${ }^{29}$ M. Lehnen, S. A. Bozhenkov, S. S. Abdullaev, M. W. Jakubowski, and the TEXTOR team, Phys. Rev. Lett. 100, 255003 (2008).

${ }^{30}$ G. Papp, M. Drevlak, T. Fülöp, and P. Helander, Nucl. Fusion 51, 043004 (2011).

${ }^{31}$ A. Wingen, S. S. Abdullaev, K. H. Finken, M. Jakubowski, and K. H. Spatschek, Nucl. Fusion 46, 941-952 (2006).

${ }^{32}$ F. Saint-Laurent, J. Bucalossi, C. Reux, S. Bremond, D. Douai, C. Gil, and P. Moreau, "Control of runaway electron beam heat loads on tore supra," in 38th EPS Conference on Plasma Physics, Straßbourg, France, 2011.

${ }^{33}$ K. H. Finken, G. Mank, A. Krämer-Flecken, and R. Jaspers, Nucl. Fusion 41, 1651-1661 (2001).
${ }^{34}$ V. Riccardo, G. Arnoux, P. Cahyna, T. C. Hender, A. Huber, S. Jachmich, V. Kiptily, R. Koslowski, L. Krlin, M. Lehnen, A. Loarte, E. Nardon, R. Paprok, D. Tskhakaya, Sr., and JET-EFDA contributors, Plasma Phys. Controlled Fusion 52, 124018 (2010).

${ }^{35} \mathrm{~S}$. Agostinelli, J. Allison, K. Amako, J. Apostolakis, H. Araujo, P. Arce, M. Asai, D. Axen, S. Banerjee, G. Barrand, F. Behner, L. Bellagamba, J. Boudreau, L. Broglia, A. Brunengo, H. Burkhardt, S. Chauvie, J. Chuma, R. Chytracek, G. Cooperman, G. Cosmo, P. Degtyarenko, A. Dell'Acqua, G. Depaola, D. Dietrich, R. Enami, A. Feliciello, C. Ferguson, H. Fesefeldt, G. Folger, F. Foppiano, A. Forti, S. Garelli, S. Giani, R. Giannitrapani, D. Gibin, J. J. Gmez Cadenas, I. Gonzlez, G. Gracia Abril, G. Greeniaus, W. Greiner, V. Grichine, A. Grossheim, S. Guatelli, P. Gumplinger, R. Hamatsu, K. Hashimoto, H. Hasui, A. Heikkinen, A. Howard, V. Ivanchenko, A. Johnson, F. W. Jones, J. Kallenbach, N. Kanaya, M. Kawabata, Y. Kawabata, M. Kawaguti, S. Kelner, P. Kent, A. Kimura, T. Kodama, R. Kokoulin, M. Kossov, H. Kurashige, E. Lamanna, T. Lampn, V. Lara, V. Lefebure, F. Lei, M. Liendl, W. Lockman, F. Longo, S. Magni, M. Maire, E. Medernach, K. Minamimoto, P. Mora de Freitas, Y. Morita, K. Murakami, M. Nagamatu, R. Nartallo, P. Nieminen, T. Nishimura, K. Ohtsubo, M. Okamura, S. O'Neale, Y. Oohata, K. Paech, J. Perl, A. Pfeiffer, M. G. Pia, F. Ranjard, A. Rybin, S. Sadilov, E. Di Salvo, G. Santin, T. Sasaki, N. Savvas, Y. Sawada, S. Scherer, S. Sei, V. Sirotenko, D. Smith, N. Starkov, H. Stoecker, J. Sulkimo, M. Takahata, S. Tanaka, E. Tcherniaev, E. Safai Tehrani, M. Tropeano, P. Truscott, H. Uno, L. Urban, P. Urban, M. Verderi, A. Walkden, W. Wander, H. Weber, J. P. Wellisch, T. Wenaus, D. C. Williams, D. Wright, T. Yamada, H. Yoshida, and D. Zschiesche, Nucl. Instrum. Methods A 506, 250-303 (2003).

${ }^{36}$ M. J. Berger, J. S. Coursey, M. A. Zucker, and J. Chang, "ESTAR database," http://www.nist.gov/pml/data/star/index.cfm, 1998.

${ }^{37}$ K. H. Finken, M. Lehnen, and S. A. Bozhenkov, Nucl. Fusion 51, 033007 (2011).

${ }^{38}$ A. Loarte, V. Riccardo, J. R. Martin-Solis, J. Paley, A. Huber, M. Lehnen, and EFDA Contributors, Nucl. Fusion 51, 073004 (2011).

${ }^{39}$ R. D. Gill, B. Alper, M. de Baar, T. C. Hender, M. F. Johnson, V. Riccardo, and Contributors to the EFDA-JET Workprogramme, Nucl. Fusion 42, 1039-1044 (2002)

${ }^{40}$ M. Lehnen, S. S. Abdullaev, G. Arnoux, S. A. Bozhenkov, M. W. Jakubowski, R. Jaspers, V. V. Plyusnin, V. Riccardo, U. Samm, JET EFDA Contributors, and the TEXTOR team, J. Nucl. Mater. 390-391, 740-746 (2009).

${ }^{41}$ T. Fülöp, H. M. Smith, and G. Pokol, Phys. Plasmas 16, 022502 (2009).

${ }^{42}$ See http://geant4.cern.ch/. 